\title{
A left main disease repeatedly treated with drug-coated balloon in a patient with poor adherence to medications
}

\author{
Song Lin Yuan ${ }^{1,2}$, Eun Jung Jun ${ }^{1}$, Moo Hyun Kim², Scot Garg ${ }^{3}$, Eun-Seok Shin ${ }^{1}$ \\ ${ }^{1}$ Department of Cardiology, Ulsan Medical Center, Ulsan, South Korea \\ ${ }^{2}$ Department of Cardiology, Dong-A University Hospital, Busan, South Korea \\ ${ }^{3}$ East Lancashire Hospitals NHS Trust, Blackburn, Lancashire, United Kingdom
}

A 45-year-old male, heavy smoker, was admitted after a 1-week history of recurrent chest pain at minimal effort. His electrocardiography, cardiac enzyme and echocardiography showed normal. Coronary angiography showed a 95\% left main (LM) stenosis with Thrombolysis in Myocardial Infarction (TIMI) grade 2 flow. He declined coronary artery bypass grafting (CABG) or stent implantation, but agreed to treatment with balloon angioplasty using a drug-coated balloon (DCB). He was carefully assessed and gave informed consent.

The lesion was dilated several times with a $3.5 \times 10 \mathrm{~mm}$ scoring balloon at $12 \mathrm{~atm}$ and then a $3.5 \times 20 \mathrm{~mm}$ DCB was inflated at $14 \mathrm{~atm}$ for $60 \mathrm{~s}$. The final angiographic result was good. His angina symptoms were resolved. He remained well until presenting 6 months later with a recurrence of his symptoms. He admitted to discontinuing all his medication, including dual antiplatelet therapy since discharge. Repeat angiography showed a 90\% restenosis at the LM with TIMI grade 3 flow. He again declined CABG or stenting. Considering his poor drug compliance, repeat revascularization to the LM with DCB by same technique was performed. Again, the final angiographic result was good and 8 months later, follow-up angiographic and intravascular ultrasound confirmed excellent results (Fig. 1). He has been well without angina for 10 months now.

This case was repeatedly treated with $\mathrm{DCB}$ given concerns regarding the patient's poor compliance to medication including antiplatelets. These findings suggest that LM disease may be a potential alternative indication for DCB treatment, especially when patients are unsuitable for long-term antiplatelet therapy or unwilling to undergo $\mathrm{CABG}$ or stenting.

Conflict of interest: None declared

Address for correspondence: Eun-Seok Shin, MD, PhD, Department of Cardiology, Ulsan Medical Center, 13, Wolpyeong-ro, 171 beon-gil, Nam-gu, Ulsan, 44686, South Korea, tel: +82-52-259-5425, fax: +82-52-259-5117,

e-mail: sesim1989@gmail.com

Received: 21.04.2020 Accepted: 5.05.2020 

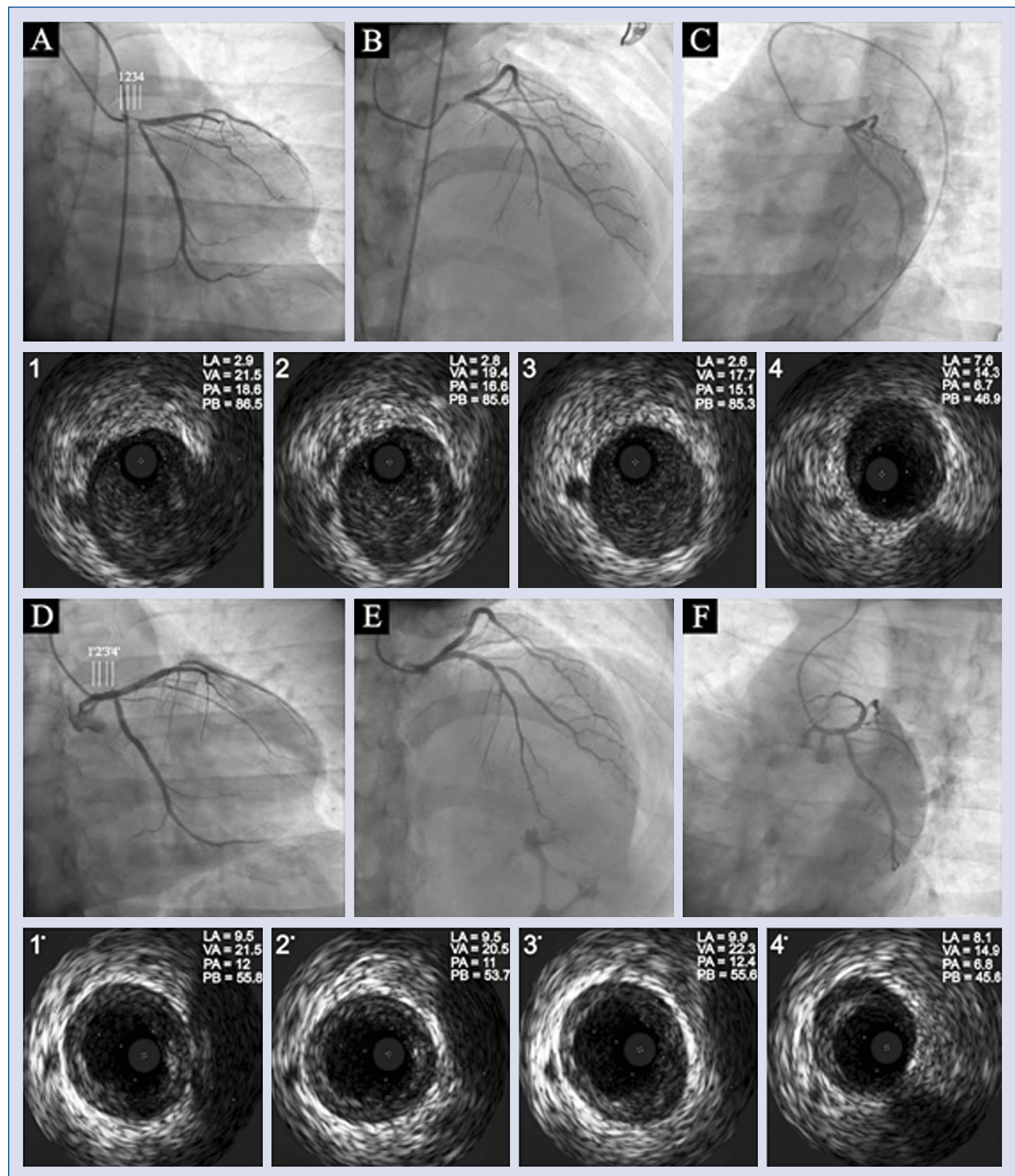

Figure 1. Pre-procedure (A-C), follow-up angiographic images (D-F) coupled to serial corresponding intravascular ultrasound (IVUS) images. At 8 months follow-up IVUS showed a much greater luminal area and decreased plaque burden in left main compared to baseline IVUS; LA — lumen area, $\mathrm{mm}^{2}$; VA — vessel area, $\mathrm{mm}^{2}$; PA — plaque area, $\mathrm{mm}^{2}$; PB - plague burden, \%. 